\title{
Effect of the antimicrobial photodynamic therapy on microorganism reduction in deep caries lesions: a systematic review and meta-analysis
}

Pâmela Oliveira Ornellas Leonardo Santos Antunes

Karla Bianca Fernandes da Costa Fontes Helvécio Cardoso Corrêa Póvoa Erika Calvano Küchler Natalia Lopes Pontes Iorio Lívia Azeredo Alves Antunes 


\title{
Effect of the antimicrobial photodynamic therapy on microorganism reduction in deep caries lesions: a systematic review and meta-analysis
}

\author{
Pâmela Oliveira Ornellas, ${ }^{\text {a }}$ Leonardo Santos Antunes, ${ }^{a}$ Karla Bianca Fernandes da Costa Fontes, ${ }^{\text {a }}$ \\ Helvécio Cardoso Corrêa Póvoa, ${ }^{b}$ Erika Calvano Küchler, ${ }^{c}$ Natalia Lopes Pontes lorio, ${ }^{b}$ and \\ Lívia Azeredo Alves Antunes ${ }^{\mathrm{a}, *}$ \\ ${ }^{a}$ Fluminense Federal University, Department of Specific Formation, School of Dentistry, Rua Doutor Silvio Henrique Braune, 22, Centro-Nova \\ Friburgo, Nova Friburgo, Rio de Janeiro, Brazil \\ ${ }^{b}$ Fluminense Federal University, Department of Basic Science, School of Dentistry, Rua Doutor Silvio Henrique Braune, 22, Centro, \\ Nova Friburgo, Rio de Janeiro, Brazil \\ 'University of São Paulo, Department of Pediatric Dentistry, School of Dentistry of Ribeirão Preto, Av. do Café, s/n - Ribeirão Preto, \\ São Paulo, Brazil
}

\begin{abstract}
This study aimed to perform a systematic review to assess the effectiveness of antimicrobial photodynamic therapy (aPDT) in the reduction of microorganisms in deep carious lesions. An electronic search was conducted in Pubmed, Web of Science, Scopus, Lilacs, and Cochrane Library, followed by a manual search. The MeSH terms, MeSH synonyms, related terms, and free terms were used in the search. As eligibility criteria, only clinical studies were included. Initially, 227 articles were identified in the electronic search, and 152 studies remained after analysis and exclusion of the duplicated studies; 6 remained after application of the eligibility criteria; and 3 additional studies were found in the manual search. After access to the full articles, three were excluded, leaving six for evaluation by the criteria of the Cochrane Collaboration's tool for assessing risk of bias. Of these, five had some risk of punctuated bias. All results from the selected studies showed a significant reduction of microorganisms in deep carious lesions for both primary and permanent teeth. The meta-analysis demonstrated a significant reduction in microorganism counts in all analyses $(p<0.00001)$. Based on these findings, there is scientific evidence emphasizing the effectiveness of aPDT in reducing microorganisms in deep carious lesions. ๑ 2016 Society of Photo-Optical Instrumentation Engineers (SPIE) [DOI: 10.1117/1.JBO.21.9 .090901]
\end{abstract}

Keywords: photochemotherapy; photodynamic therapy; dental caries.

Paper 160389VR received Jun. 9, 2016; accepted for publication Aug. 31, 2016; published online Sep. 21, 2016.

\section{Introduction}

Dental caries remains one of the most prevalent chronic diseases in humans, and it is an important cause of oral pain and dental loss, which lead to school and work absenteeism, affecting individuals' daily activities, and emotional stability. ${ }^{1}$ Dental caries is caused by specific pathogenic microorganisms that metabolize carbohydrates ingested to form acids, deficient oral hygiene habits, and high-sugar diets. ${ }^{2}$

Growing evidence indicates that one-step incomplete excavation seems suitable to treat deep caries lesions. ${ }^{3}$ The partial removal of carious tissue and the subsequent restoration of the dental element are sufficient to reduce the caries microbiota. ${ }^{4}$ Protocols for ultraconservative caries removal, such as Carisolv ${ }^{\mathrm{TM}^{5}}$ and Papacarie ${ }^{\mathrm{TM}},{ }^{6}$ have been used as an alternative method for complementing the effects of manual excavation, thus reducing cariogenic microbiota.

Several studies have shown that oral bacteria are susceptible to antimicrobial photodynamic therapy (aPDT)., ${ }^{7,8}$ aPDT has recently been studied as a coadjuvant therapy against microorganisms of dental caries, which suggests that it might be useful as adjunctive therapy to current deep carious lesion. ${ }^{9-14}$ Therefore,

*Address all correspondence to: Lívia Azeredo Alves Antunes, E-mail: liviaazeredo@gmail.com the aim of this study was to perform a systematic review and meta-analysis to evaluate the effect of aPDT as a coadjuvant therapy in reducing microorganisms in deep carious lesions.

\section{Methods}

This systematic review was registered in the PROSPERO database (PROSPERO registry number: CRD42015029891) and was conducted following the PRISMA statements. ${ }^{15}$

\subsection{Focused Question}

Is aPDT an effective coadjuvant therapy to reduce microorganisms in deep carious lesions?

\subsection{Strategy for Identification and Selection of the Studies}

A broad search for articles was conducted, and only articles published before March 29, 2016, were considered for review. The selection process is described in Fig. 1. The following databases were used: Pubmed, Web of Science (WOS), Scopus, Cochrane Library, and Lilacs. The gray literature was also consulted trough Opensigle. The MeSH terms "Photochemotherapy" and "Dental caries" were used. MeSH synonyms, related terms, and free terms were included. These keywords were selected 


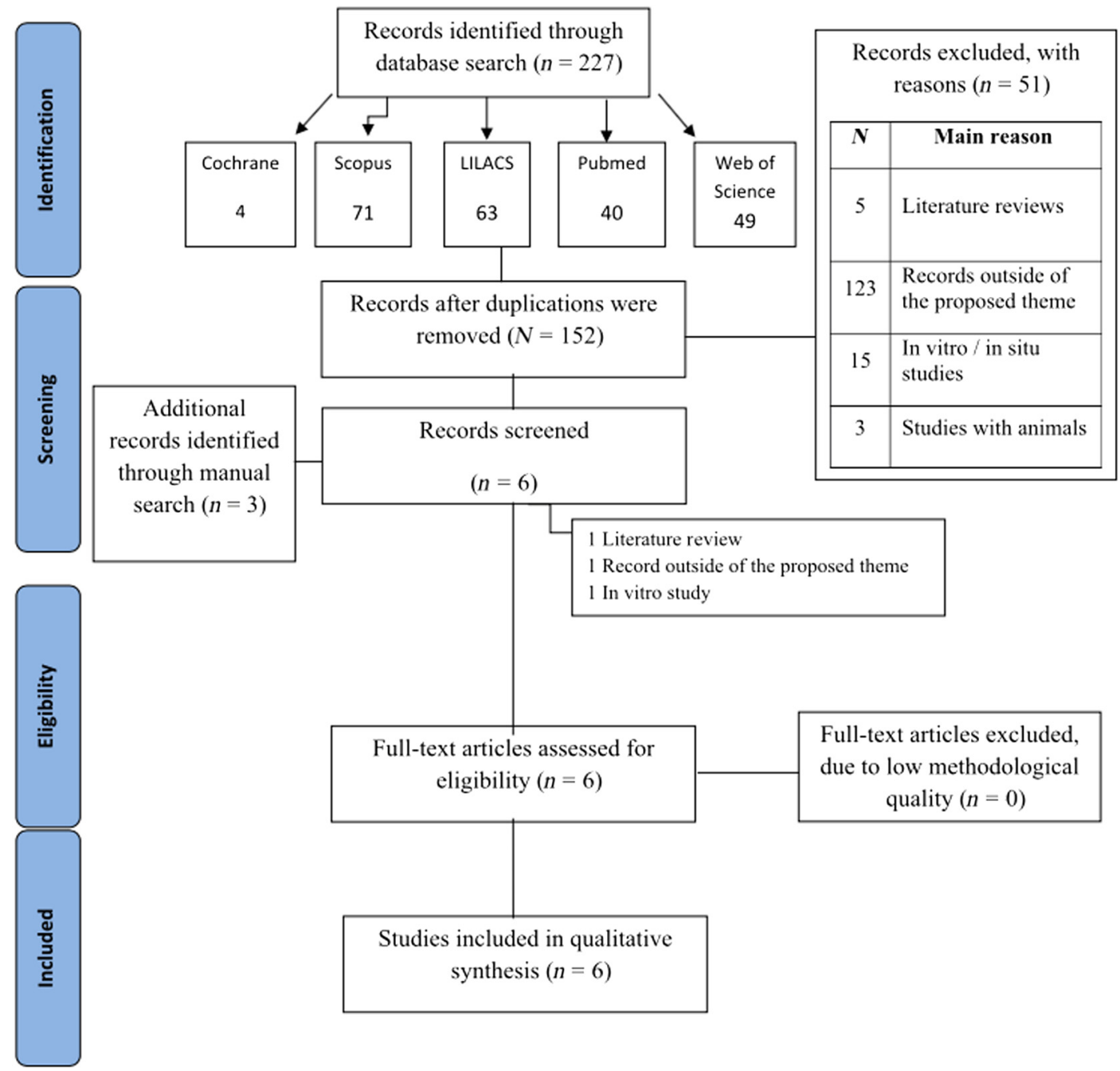

Fig. 1 Flowchart for process of article search and selection using the preferred reporting items for systematic reviews (PRISMA) framework.

from DeCS—Health Sciences Descriptors developed and from the Medical Subject Headings (MeSH) of the US National Library of Medicine. The terms were combined to refine the search results (the combination of these search descriptors is shown in Table 1). The titles and abstracts of the identified articles were independently evaluated by two researchers (POO and LAA) to determine whether they met the inclusion criteria for the review. The electronic search was supplemented by a manual search of the reference list from the included articles.

\subsection{Eligibility Criteria}

The publications were selected if they were in vivo clinical studies. There was no restriction on language. The inclusion criteria outlines articles according to the population, intervention, comparisons, and outcomes (PICOS) as follows:

Population (P): Healthy humans who were not taking any medication that could affect the results of the study.

Intervention (I): Treatment of deep carious lesions.

Comparison (C): Group treated with aPDT and group treated without aPDT; and/or comparison before and after aPDT.
Outcome (O): microorganism count.

Study design (S): clinical studies.

Duplicate articles, literature reviews, records out of the proposed theme, case reports, in vitro studies, dissertations, thesis, or monographs, and studies with animals were excluded.

\subsection{Study Selection}

Initially, two of the authors (POO and LAA) selected the studies by title and abstracts according to the previously described search strategy (PICOS criteria). To evaluate agreement between authors, $10 \%$ of the publications were randomly selected and had their classification compared, and then a Kappa statistic of 0.97 was determined. Only articles that matched the inclusion criteria were accepted. Articles appearing in more than one database were considered only once (Fig. 1). Subsequently, the full texts of the potentially eligible studies were completely accessed and the PICOS criteria were applied again. Any disagreement was discussed and solved by consensus or discussion with the third review author. After the inclusion of the abstracts that fulfilled the selection criteria and verification of eligibility by reading the complete articles, the articles were submitted to verify the quality assessment and risk of bias. 
Table 1 Electronic database used and search strategy.

\begin{tabular}{|c|c|}
\hline Database & Search strategy \\
\hline PubMed & $\begin{array}{l}\text { \#1 ("Photochemotherapy" [MeSH Terms] OR } \\
\text { "Photochemotherapy" [Title/Abstract] OR } \\
\text { "Photodynamic Therapies" [Title/Abstract] OR } \\
\text { "Photodynamic Therapy" [Title/Abstract] OR "TFD" } \\
\text { [Title/Abstract]) } \\
\text { \#2 ("Dental Caries" [MeSH Terms] OR "Dental } \\
\text { Caries" [Title/Abstract] OR "Dental Decay" [Title/ } \\
\text { Abstract] OR "Carious Dentin" [Title/Abstract] OR } \\
\text { "Carious Dentins" [Title/Abstract]) } \\
\text { \#1 and \#2 }\end{array}$ \\
\hline Scopus & $\begin{array}{l}\text { (TITLE-ABS-KEY (Photochemotherapy) OR TITLE- } \\
\text { ABS-KEY (Photodynamic Therapies) OR TITLE- } \\
\text { ABS-KEY (Photodynamic Therapy OR TITLE-ABS- } \\
\text { KEY (TFD)) } \\
\text { (TITLE-ABS-KEY (Dental Caries) OR TITLE-ABS- } \\
\text { KEY (Dental Decay) OR TITLE-ABS-KEY (Carious } \\
\text { Dentin) OR TITLE-ABS-KEY (Carious Dentins)) } \\
\# 1 \text { and \#2 }\end{array}$ \\
\hline wOS & $\begin{array}{l}\text { \#1 TS=(“Photochemotherapy" OR "Photodynamic } \\
\text { Therapies" OR "Photodynamic Therapy" OR "TFD") } \\
\text { \#2 TS=(“Dental Caries" "OR "Dental Decay" OR } \\
\text { "Carious Dentin" OR "Carious Dentins") } \\
\text { \#1 and \#2 }\end{array}$ \\
\hline Lilacs & $\begin{array}{l}\text { (ab:(Photochemotherapy)) OR (ab:(Photodynamic } \\
\text { Therapies)) OR (ab:(Photodynamic Therapy)) OR } \\
\text { (ab:(TFD)) AND (ab:(Dental Caries)) OR (ab:(Dental } \\
\text { Decay)) OR (ab:(Carious Dentin)) OR (ab:(Carious } \\
\text { Dentins)) }\end{array}$ \\
\hline $\begin{array}{l}\text { Cochrane } \\
\text { Library }\end{array}$ & $\begin{array}{l}\# 1 \text { (Dental Caries:ti,ab,kw or Dental Decay:ti,ab,kw } \\
\text { or Carious Dentin:ti,ab,kw or Carious Dentins:ti,ab, } \\
\text { kw) } \\
\text { \#2 (Photochemotherapy:ti,ab,kw or Photodynamic } \\
\text { Therapy:ti,ab,kw or Photodynamic Therapies:ti,ab, } \\
\text { kw or TFD:ti,ab,kw) } \\
\text { \#1 and \#2 }\end{array}$ \\
\hline
\end{tabular}

\subsection{Assessment of Risk of Bias in Included Studies}

We used the Cochrane Collaboration's tool for assessing risk of bias. ${ }^{16}$ The following domains were assessed: generation of allocation sequence, allocation concealment, blinding of participants and outcome assessors, incomplete outcome data, and selective outcome reporting. Each domain was classified as having low (+), high (-), or uncertain (?) risk of bias. The authors also included studies with no description of inclusion and exclusion criteria as a possible risk of bias.

\subsection{Data Synthesis}

The data from the included papers were compiled. Data extraction was conducted independently by two reviewers (LAA and POO) by completely reading the articles and considering the categories and variables. We considered the following clinical parameters: sample/number of teeth, age, dentition/teeth, groups assessed, irradiation location, and removal of photosensitizer (PS) before irradiation. We also evaluated the aPDT parameters: PS type, concentration, and preirradiation time, active laser media, laser wavelength $(\mathrm{nm})$, energy $(\mathrm{J})$, dose $\left(\mathrm{J} / \mathrm{cm}^{2}\right)$, power $(\mathrm{mW})$, irradiance $\left(\mathrm{mW} / \mathrm{cm}^{2}\right)$, spot size $\left(\mathrm{mm}^{2}\right)$, and duration of irradiation $(\mathrm{min})$.

\subsection{Meta-Analysis}

A meta-analysis was also performed to combine comparable results. The outcome was presented in all studies as continuous data. For the meta-analysis, we extracted the mean and the standard deviation ( $\log 10 \mathrm{CFU} / \mathrm{ml})$. Subgroups were established prior to the overall analysis of the outcome according to the evaluated microorganism(s) as follows: 1) total viable microorganisms counts; 2) mutans streptococci counts; and 3) Lactobacillus spp. counts.

A fixed effect model was used for the meta-analysis. The weighted standard mean differences before and after aPDT were performed using the inverse-variance meta-analysis. Publication bias was assessed using a funnel plot. The $I^{2}$ was used to assess statistical heterogeneity between studies, where $I^{2}$ values of $25 \%, 50 \%$, and $75 \%$ indicated low, medium, and highheterogeneity, respectively. ${ }^{17}$ The meta-analysis calculation and Forest plots creations were performed with RevMan 5.3. In the Forest plots, negative values in the mean difference represent an increase in microorganism count.

\section{Results}

\subsection{Search and Selection of Articles}

A flow diagram of the search strategy is presented in Fig. 1. Initially, the search resulted in 227 published studies: 40 from PubMed, 4 from Cochrane, 71 from Scopus, 63 from LILACS, 49 from WOS, and 0 from Opensigle. Seventy-five records were excluded because they were duplicated. The analysis of the titles and abstracts resulted in the exclusion of 146 of the published studies, leaving 6 for full text reading. The references of the six remaining articles were hand searched and more three articles were selected. This resulted in a total of nine articles selected for reading in full. After access to the complete file, three records were excluded because one was a literature review, another because it was outside of the proposed theme, and the last was an in vitro study, thus leaving only six articles.

\subsection{Quality Assessment of Risk of Bias}

Figure 2 demonstrates the evaluation of the inner methodological risk of bias, according to the Cochrane Collaboration's tool for assessing risk of bias. Melo et al.'s ${ }^{13}$ study presented the best classification. In general, the results and exclusion criteria were well described in all selected papers. The risk of bias was more frequent in allocation, randomization, and sample size calculation.

\subsection{Clinical Parameters}

Data extraction from the selected articles is described in Table 2. In the included articles, the sample size ranged from 10 to 90 teeth. Of the six selected articles, only Longo et al. ${ }^{11}$ did not provide the patient age. One article ${ }^{12}$ did not 


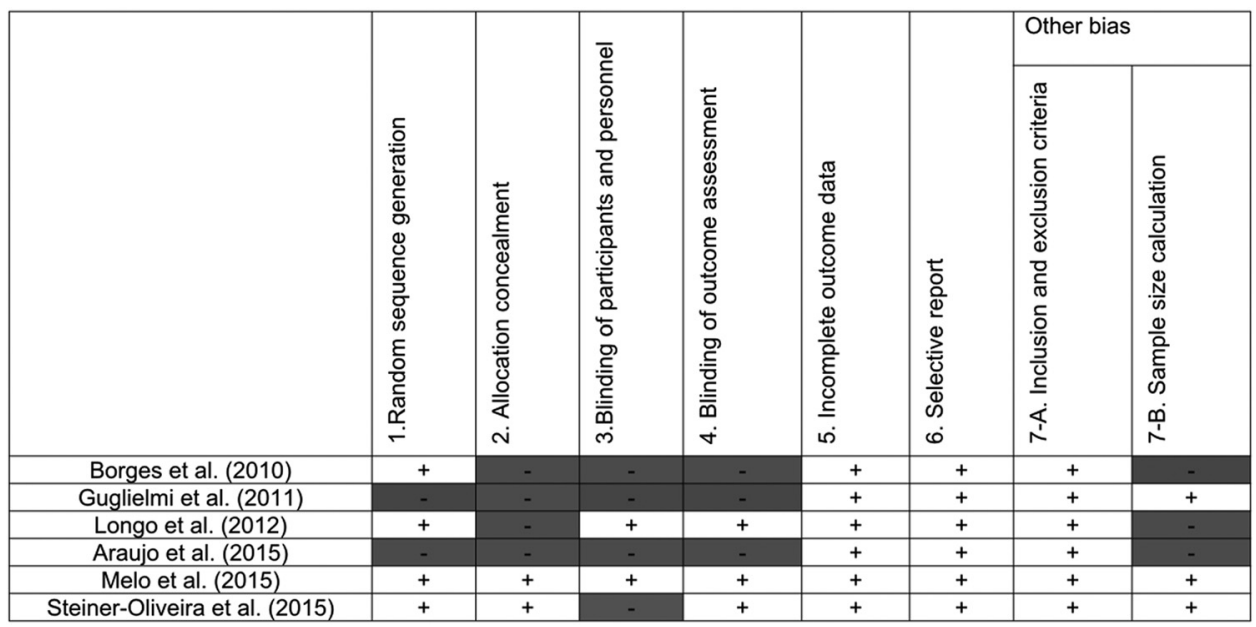

Fig. 2 Quality assessment of the selected studies (the cochrane collaboration tool for assessing risk of bias). Yes (+) -low risk of bias; No (-) -high risk of bias. The cells shaded in gray present higher the risk of bias.

Table 2 Characteristics of the included studies.

\begin{tabular}{|c|c|c|c|c|c|c|}
\hline $\begin{array}{l}\text { Author/ } \\
\text { year }\end{array}$ & Type of study & $\begin{array}{l}\text { Sample/ } \\
\text { number of } \\
\text { teeth }\end{array}$ & Age & $\begin{array}{l}\text { Dentition/ } \\
\text { teeth }\end{array}$ & Groups evaluated & Outcome \\
\hline $\begin{array}{l}\text { Borges } \\
\text { et al., } \\
2010\end{array}$ & Case-control & $5 / 20$ & $\begin{array}{c}19 \text { to } \\
36 \text { years }\end{array}$ & $\begin{array}{l}\text { Permanent } \\
\text { MD }\end{array}$ & $\begin{array}{l}\text { 1. Without TBO and without light } \\
\text { 2. With TBO alone } \\
\text { 3. With aPDT alone } \\
\text { 4. With TBO plus aPDT }\end{array}$ & $\begin{array}{l}\text { The association of TBO and aPDT } \\
\text { was effective in killing oral } \\
\text { microorganisms present in carious } \\
\text { dentin lesions }\end{array}$ \\
\hline $\begin{array}{l}\text { Guglielmi } \\
\text { et al., } \\
2011\end{array}$ & Case-control & $23 / 26$ & $\begin{array}{c}8 \text { to } \\
25 \text { years }\end{array}$ & $\begin{array}{l}\text { Permanent } \\
\text { /molars }\end{array}$ & $\begin{array}{l}\text { In the same teeth, they were } \\
\text { compared before and immediately } \\
\text { after aPDT mediated by MB }\end{array}$ & $\begin{array}{l}\text { aPDT may be an appropriate } \\
\text { approach for the treatment of deep } \\
\text { carious lesions using minimally } \\
\text { invasive procedures }\end{array}$ \\
\hline $\begin{array}{l}\text { Longo } \\
\text { et al., } \\
2012\end{array}$ & Case-control & $10 / 12$ & $\begin{array}{l}\text { Children } \\
\text { and adults }\end{array}$ & $\begin{array}{l}\text { Primary and } \\
\text { permanent/ } \\
\text { molars }\end{array}$ & $\begin{array}{l}\text { In the same teeth, they were } \\
\text { compared before and immediately } \\
\text { after aPDT mediated by AICIPC } \\
\text { liposomal solution }\end{array}$ & $\begin{array}{l}\text { aPDT protocol mediated by } \\
\text { cationic liposomes containing } \\
\text { AICIPc is safety for clinical } \\
\text { application and is efficient in the } \\
\text { reduction of bacterial load in caries } \\
\text { lesions }\end{array}$ \\
\hline $\begin{array}{l}\text { Araújo } \\
\text { et al., } \\
2015\end{array}$ & Case-control & WD/10 & $\begin{array}{c}3 \text { to } \\
9 \text { years }\end{array}$ & WD/Molars & $\begin{array}{l}\text { Using the same teeth, the following } \\
\text { groups were assessed: } \\
\text { 1. Untreated superficial dentin } \\
\text { 2. Untreated deep dentin } \\
\text { 3. aPDT-treated deep dentin } \\
\text { directly irradiated } \\
\text { 4. aPDT-treated superficial dentin } \\
\text { 5. aPDT-treated deep dentin not } \\
\text { directly irradiated }\end{array}$ & $\begin{array}{l}\text { Although aPDT may not affect the } \\
\text { number of } S \text {. mutans DNA copies } \\
\text { immediately after the treatment, } \\
\text { clear reduction of the number of } \\
\text { CFU was found }\end{array}$ \\
\hline $\begin{array}{l}\text { Melo } \\
\text { et al., } \\
2015\end{array}$ & $\begin{array}{l}\text { Single blind, } \\
\text { randomized, } \\
\text { controlled, split- } \\
\text { mouth, clinical trial }\end{array}$ & $45 / 90$ & $\begin{array}{c}\text { Above } \\
18 \text { years }\end{array}$ & $\begin{array}{l}\text { Permanent } \\
\text { /posterior } \\
\text { teeth }\end{array}$ & $\begin{array}{l}\text { 1. Control group: } 0.89 \% \mathrm{NaCl} \\
\text { 2. Experimental group: aPDT } \\
\text { mediated by TBO }\end{array}$ & $\begin{array}{l}\text { aPDT significantly reduced viable } \\
\text { counts of all tested } \\
\text { microorganisms }\end{array}$ \\
\hline $\begin{array}{l}\text { Steiner- } \\
\text { Oliveira } \\
\text { et al., } \\
2015\end{array}$ & Clinical trial & $32 / W D$ & $\begin{array}{c}5 \text { to } \\
7 \text { years }\end{array}$ & $\begin{array}{l}\text { Primary/ } \\
\text { molars }\end{array}$ & $\begin{array}{l}\text { 1. Control: } 2 \% \text { chlorhexidine; } \\
\text { 2. Group LEDTB:aPDT with LED } \\
\text { (LED) mediated by TBO; } \\
\text { 3. Group LMB: aPDT with laser } \\
\text { mediated by MB }\end{array}$ & $\begin{array}{l}\text { Independent of the treatment, a } \\
\text { significant reduction in } \\
\text { microorganisms was found for } \\
\text { universal bacteria }\end{array}$ \\
\hline
\end{tabular}

Note: Abbreviations: MB, methylene blue; TBO, toluidine blue; aPDT, antimicrobial photodynamic therapy; AICIPc, aluminum-chloride-phthalocyanine; WD, without data. 
Ornellas et al.: Effect of the antimicrobial photodynamic therapy on microorganism reduction...

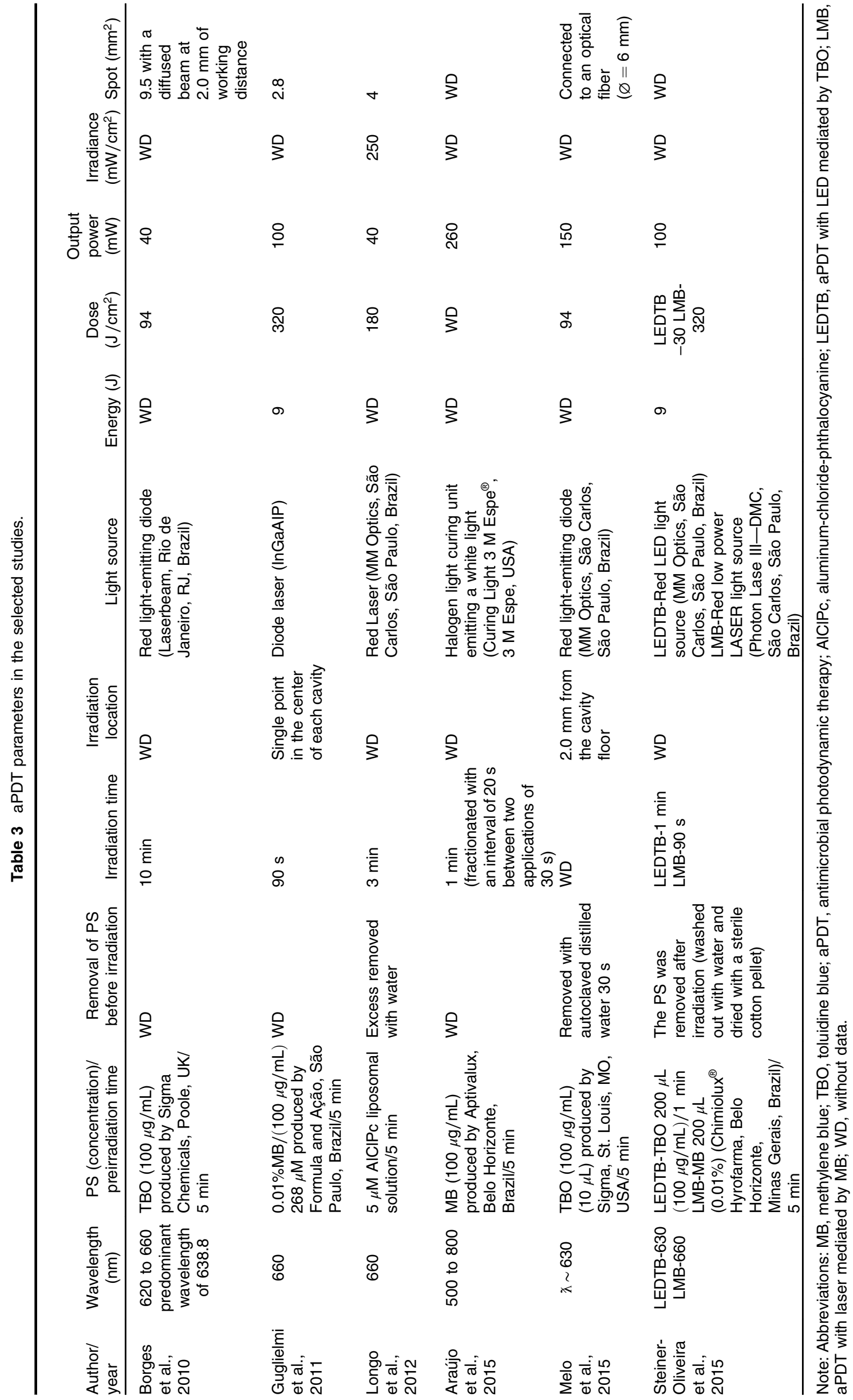


Ornellas et al.: Effect of the antimicrobial photodynamic therapy on microorganism reduction...

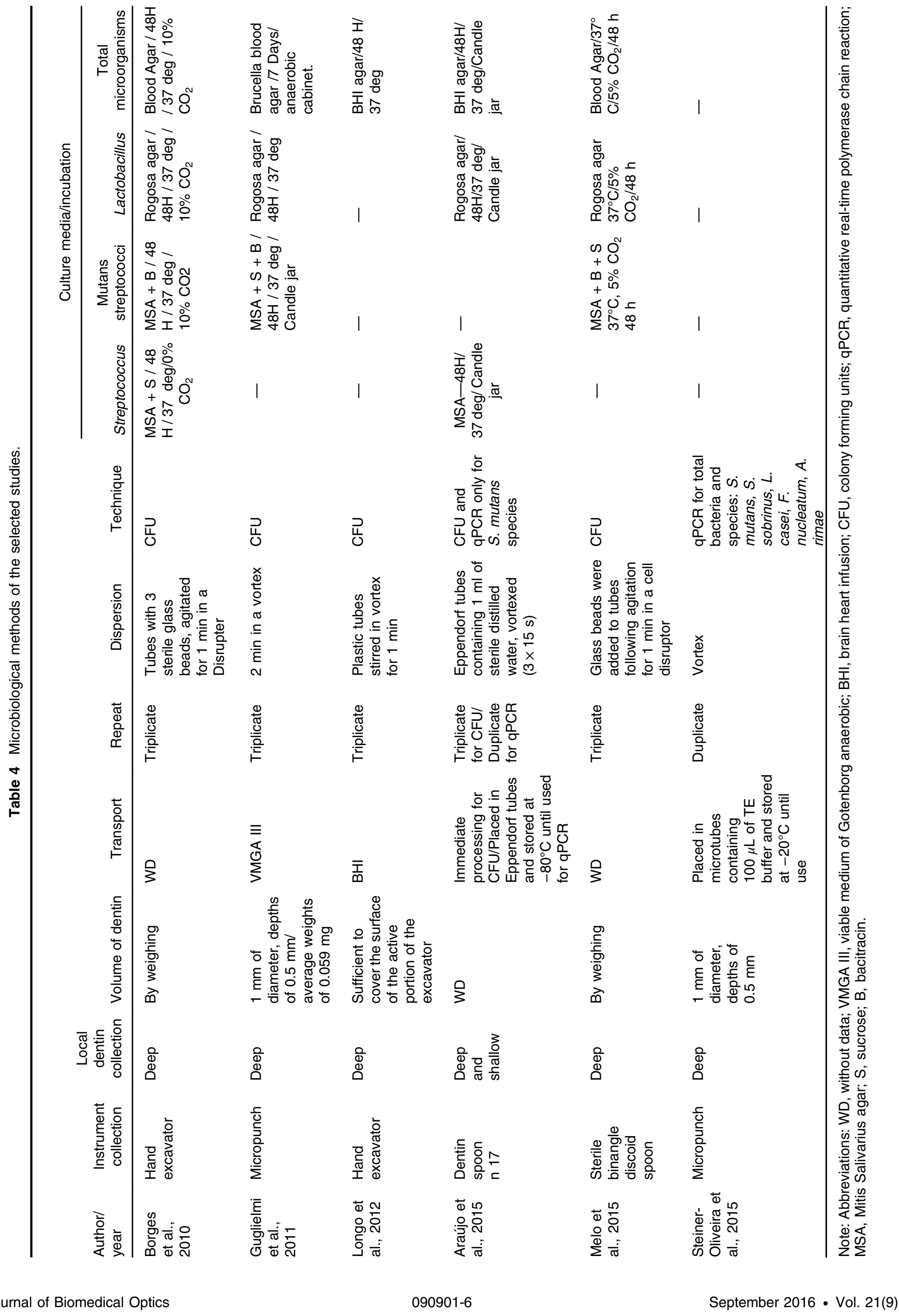


describe the type of teeth used. Three articles ${ }^{9,10,13}$ used permanent teeth, another ${ }^{11}$ used primary and permanent molars, and only one of the selected articles used primary molars. ${ }^{14}$ Five articles ${ }^{9-12,14}$ used the same teeth after manual excavation and compared before (control) and immediately after aPDT (experimental). Only Melo et al. ${ }^{13}$ performed a randomized, controlled, intraindividual comparison (split-mouth), with two groups (intervention and control).

\section{4 aPDT Parameters}

The data collected from the six selected studies regarding the aPDT parameters are summarized in Table 3. The laser wavelength $(\mathrm{nm})$ used ranged between 500 and 800 and the diameter of the spot the laser focused on varied between 2.8 and $9.5 \mathrm{~mm}^{2}$. The output power ranged between 40 and $260 \mathrm{~mW}$ and the duration of irradiation ranged between 1 and $10 \mathrm{~min}$. Melo et al. ${ }^{13}$ did not describe the irradiation duration. The PSs used included toluidine blue (TBO), methylene blue (MB), and aluminumchloride-phthalocyanine (AlClPc). Guglielmi et al. ${ }^{10}$ and Araújo et al. ${ }^{12}$ used MB as the PS in their studies. TBO was used as PS in studies by Borges et al., ${ }^{9}$ and Melo et al. ${ }^{13}$ Longo et al. ${ }^{11}$ used AlClPc, whereas Steiner-Oliveira et al. ${ }^{14}$ evaluated two PSs (TBO and MB). Guglielmi et al. ${ }^{10}$ and Longo et al. ${ }^{11}$ used only one group treated with aPDT, whereas Borges et al., ${ }^{9}$ Araújo et al., ${ }^{12}$ Melo et al., ${ }^{13}$ and Steiner-Oliveira et al. ${ }^{14}$ divided the sample into different groups receiving different treatment alternatives: aPDT with TBO, PDT with MB, and chlorhexidine (Table 2).

\subsection{Microbiological Analyses}

According to the information described in Table 4, we concluded that most studies performed the collection of deep dentin, except Araújo et al., ${ }^{12}$ which completed the collection of both deep dentin and shallow dentin as well. Regarding the form of collection, most authors used manual resource diggers. Guglielmi et al. ${ }^{10}$ and Steiner-Oliveira et al. ${ }^{14}$ differed by using a micropunch.

With respect to the volume of dentin collected, Borges et al. ${ }^{9}$ and Melo et al. ${ }^{13}$ quantified this volume by weight. Araújo et al. ${ }^{12}$ did not provide this information. Guglielmi et al. ${ }^{10}$ mentioned an average weight, whereas Longo et al. ${ }^{11}$ described the collected dentin only as a volume sufficient to cover the surface of the active portion of the excavator. Steiner-Oliveira et al. ${ }^{14}$ and Guglielmi et al. ${ }^{10}$ reported doing the dentin collection using a micropunch and gave details on the diameter and penetration of the instrument, but only Guglielmi et al. ${ }^{10}$ measured the average weight of the dentin.

In most studies, the gold standard method for detecting the antimicrobial effect of aPDT - the conventional culture method by CFU—was used. Borges et al. ${ }^{9}$ evaluated colonies of mutans streptococci, total streptococci, lactobacilli, and total microorganisms. Guglielmi et al. ${ }^{10}$ and Melo et al. ${ }^{13}$ did not take a count Streptococcus, Araújo et al. ${ }^{12}$ did not evaluate colonies of mutans streptococci, and Longo et al. ${ }^{11}$ determined only the total viable bacteria. Araújo and coworkers ${ }^{12}$ used the real-time PCR to detect Streptococcus mutans species, and Steiner-Oliveira et al. ${ }^{14}$ also used the real-time PCR to assess the total bacteria and five specific species ( $S$. mutans, Streptococcus sobrinus, Lactobacillus casei, Fusobacterium nucleatum, and Atopobium rimae). The studies that used the

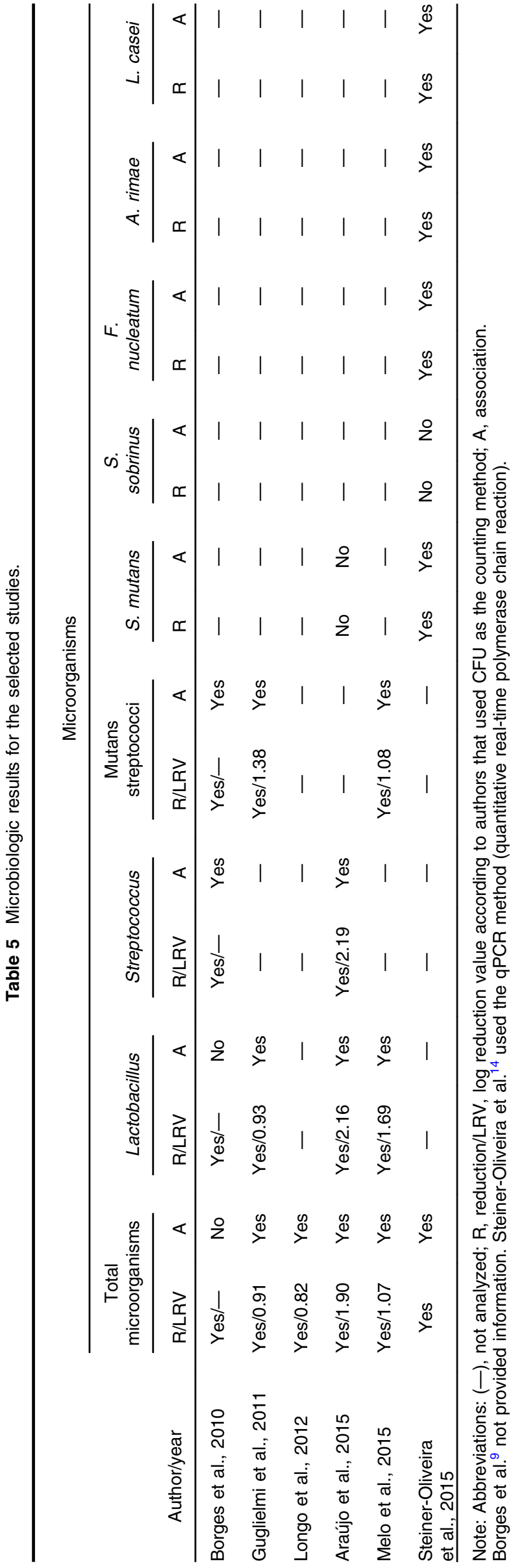


CFU method were run in triplicate, while the ones that choose PCR performed in duplicate

As described in Table 5, all studies observed CFU reduction for all kinds of microorganisms evaluated by this method. However, Araújo et al. ${ }^{12}$ also used the real-time PCR analyses and showed that the $S$. mutans DNA did not reduce after aPDT, whereas Steiner-Oliveira et al. ${ }^{14}$ demonstrated that aPDT evaluated by real-time PCR analyses reduced the total number of total bacteria, $S$. mutans, $S$. sobrinus, $F$. nucleatum, A. rimae after each treatment, but did not reduce $S$. sobrinus.

\subsection{Meta-Analysis}

Two articles were not included in the meta-analysis: one article 9 due the fact that there was missing data, and we did not achieve contact with the authors, and the other ${ }^{14}$ only used real-time PCR to analyze the microorganism reduction. The four included studies evaluated the total viable bacteria count. ${ }^{10-13}$ Three studies $^{10,12,13}$ evaluated Lactobacillus spp. counts and two studies ${ }^{10,13}$ evaluated mutans streptococci counts. Forest plots are presented in Fig. 2. The studies showed low heterogeneity in the total viable bacteria counts analysis and in the mutans streptococci counts analysis $\left(I^{2}=0 \%\right)$. The Forest plots (Fig. 3) demonstrated that all of the meta-analyses presented a significant difference before and after aPDT. For the total viable bacteria count analyses, mean difference and 95\% confidence interval was $1.32[1.13,1.51] ;(p<0.00001)$. Mean difference and $95 \%$ confidence interval for mutans streptococci counts were $1.63[1.05,2.20] ;(p<0.00001)$ and were 1.50 [1.22, 1.79]; $(p<0.00001)$ for Lactobacillus spp.

\section{Discussion}

aPDT has been gaining attention in research on alternative antimicrobial approaches, and studies have shown that aPDT has such properties. ${ }^{18,12,19}$ aPDT is effective in killing oral microorganisms present in dentine caries that are produced in situ and in vitro and may be useful in minimally invasive dentistry. ${ }^{20,21}$ Assuming that the in vitro and in situ techniques are effective, we suggest the following question: Is aPDT an effective coadjuvant therapy to reduce microorganisms in deep carious lesions? To answer this question, a systematic review and meta-analysis were carried out.

Systematic reviews and meta-analyses are important tools and are commonly used for scientific evidence of health practices. They are likely to be used with increasing frequency as current initiatives to share clinical trial data gain momentum and may be particularly important in reviewing controversial therapeutic areas. ${ }^{15}$ These types of studies also provide possible recommendations for future studies, evaluate the applied research methods, and provide a summary of evidence related to a specific intervention strategy.

Our systematic review and meta-analysis of the included studies clearly supported the hypothesis that aPDT is an effective coadjuvant tool in reducing microorganisms in the treatment of deep carious lesions. A significant reduction in microorganism counts was observed in all analysis, which highlights this method as a useful minimally invasive tool for dental clinicians.

The included studies used different clinical protocols. Longo et al., ${ }^{11}$ Araújo et al., ${ }^{12}$ and Steiner-Oliveira et al. ${ }^{14}$ did not describe the location point where the irradiation was carried

(a)

\begin{tabular}{lrrrrr} 
(a) & \multicolumn{3}{c}{ Before PDT } & \multicolumn{2}{c}{ After PDT } \\
Study or subgroup & Mean & SD & Total & Mean & SD T T \\
\hline Araujo et al. (2015) & 5.55 & 0.14 & 10 & 3.21 & 0.5 \\
Guglielmi et al. (2011) & 7.1 & 0.72 & 26 & 6.19 & 1.38 \\
Longo et al. (2012) & 3.42 & 0.24 & 12 & 2.6 & 0.44 \\
Melo et al. (2015) & 5.1 & 1.2 & 45 & 4.45 & 1.3 \\
& \multicolumn{4}{c}{93} \\
Total (95\% CI) \\
Heterogeneity: Chi ${ }^{2}=58.79, \mathrm{df}=3(\mathrm{P}<0.00001) ; I^{2}=95 \%$ \\
Test for overall effect: $\mathrm{Z}=13.83(\mathrm{P}<0.00001)$
\end{tabular}

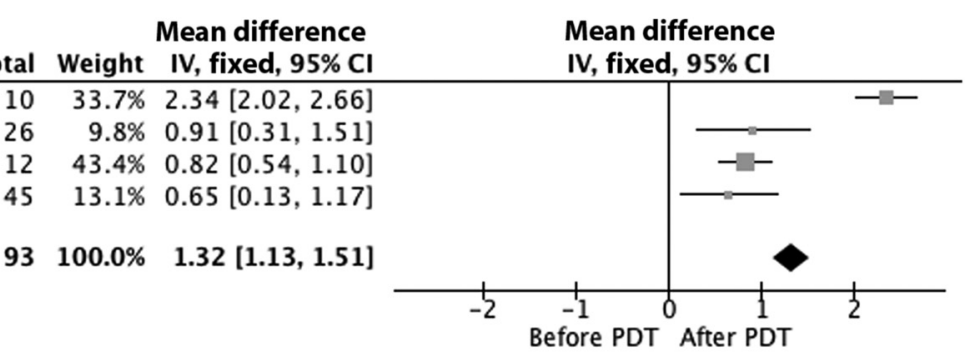

(b)

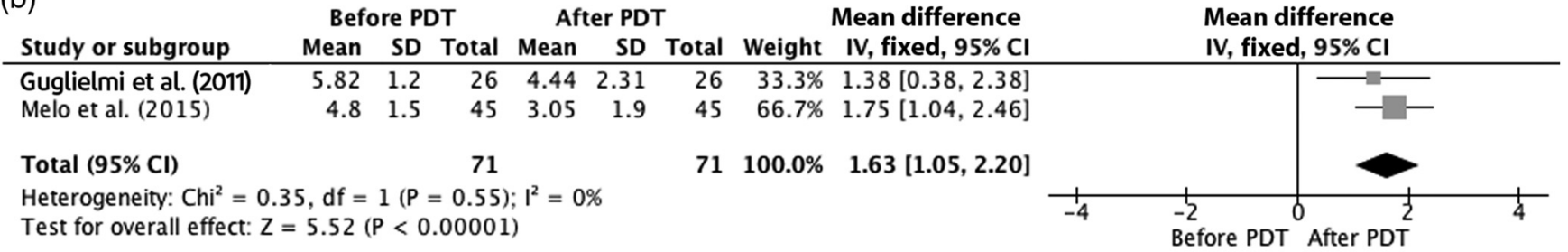

(c)

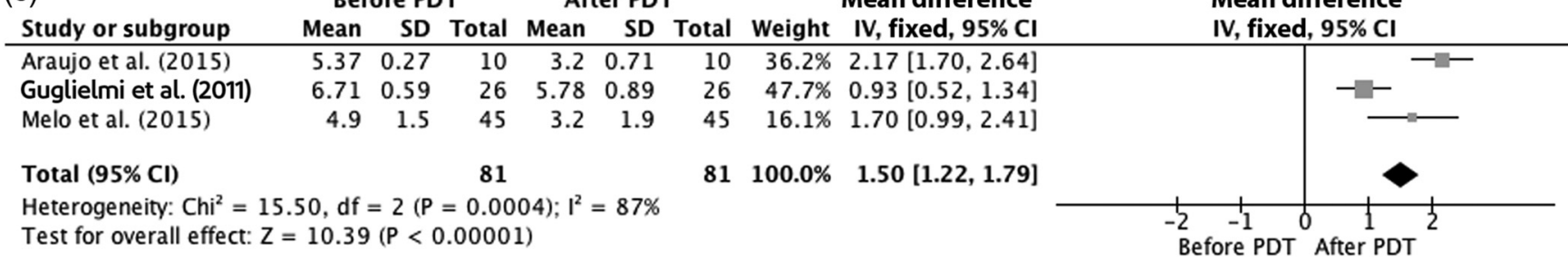

Fig. 3 Mean and the standard deviation (Log $10 \mathrm{CFU} / \mathrm{ml}$ ) comparison before and after aPDT regarding established subgroups: (a) total viable bacteria; (b) mutans streptococci; and (c) Lactobacillus spp. counts. 
out. Araújo et al. ${ }^{12}$ held a 1-min irradiation, divided into two irradiations of $30 \mathrm{~s}$. However, it is not described whether or not irradiation was performed at a single point or two points in the cavity. The same author made an analysis of superficial and deep dentin, but the result when comparing to control was statistically different when the dentin was directly irradiated or not directly irradiated. Thus, it is suggested that new studies should evaluate if the irradiation at more than one point in the cavity results in a greater reduction of microorganisms or, if a single point of irradiation in the cavity is enough. The single irradiation point would be very beneficial, especially for treatment of primary teeth in pediatrics, because the shorter the chair time, the better. Furthermore, three of the six studies did not mention the removal of excess PS. Longo et al. ${ }^{11}$ and Melo et al. ${ }^{13}$ removed the excess before the irradiation, whereas Steiner-Oliveira et al. ${ }^{14}$ carried out the irradiation first, followed by the removal of PS excess.

In some studies, the reported data were confusing. Longo et al. ${ }^{11}$ used adults and children in their sample, however, they did not report the findings in each age group separately. Araújo et al. ${ }^{12}$ used a sample of children aged from 3 to 9-years old, however, it was not clear what type of teeth were studied since the children in this age group can present deciduous or permanent molars. This lack of information in both studies can raise questions such as what kind of teeth is aPDT more effective for, deciduous or permanent? Could the primary dentition respond to the treatment differently than the permanent dentition, and thus have different results? Based on these questions, we recommend future studies to confirm or refute these hypotheses.

The six evaluated studies presented potential risk of bias. Only Melo et al. ${ }^{13}$ followed all checklist recommendations reported by the CONSORT statement (consolidated standards of reporting trials). ${ }^{22}$ Only three studies supported their findings on sample size calculation. ${ }^{10,13,14}$ From the six selected and analyzed studies, four underwent randomization..$^{9,11,13,14}$ Regarding the blinding of participants (single blind), Longo et al., ${ }^{11}$ Melo et al., ${ }^{13}$ and Steiner-Oliveira et al. ${ }^{14}$ followed this CONSORT recommendation for conducting microbiology analyses using blind counting of codified labeled samples (double blind) ensuring the absence of biased results.

From analyzing the aPDT parameters, we observed that the authors used different parameters, such as PS, light source, and dosimetry. Borges et al. ${ }^{9}$ and Melo et al., ${ }^{13}$ used red light-emitting diode (LED) as a light source. Steiner-Oliveira et al. ${ }^{14}$ also used LED as a light source but only in the group of PS TBO (LEDTB). The light source is selected according to the type of PSs. In terms of costs, LED present advantages when compared with the laser sources. A difference in irradiation time was also used. However, all studies achieved positive results in reducing bacteria even while using different application times. This observation suggests that a protocol with a shorter exposure period would be highly advantageous. In the case of pediatric care, this would be a wonderful advantage as shortened chair time is a priority.

Despite the fact that the different aPDT parameters have been applied in different ways, all of the studies reached a reduction of most of the tested microorganisms, thus confirming the effectiveness of the use of aPDT as an adjunctive in the treatment of deep caries. However, among the observed reductions, some studies showed significant reductions and others did not, which may have been influenced by different parameters of
aPDT. Guglielmi et al. ${ }^{10}$ and Araújo et al. ${ }^{12}$ used MB as a PS in their clinical protocol, and both placed the PS in contact with the teeth for $5 \mathrm{~min}$ before irradiation. Although these authors used different light sources, their results were similar.

Borges et al. ${ }^{9}$ and Melo et al., ${ }^{13}$ in turn, used the same PS (TBO), the same light source, red LED, and the same dose of $94 \mathrm{~J} / \mathrm{cm}^{2}$, but they used different output powers. Borges et al. ${ }^{9}$ used the output power of $40 \mathrm{~mW}$, whereas Melo et al. ${ }^{13}$ used $150 \mathrm{~mW}$. Thus, these different parameters used in the aPDT protocol may have influenced the difference in results for the species present in total microorganisms and for Lactobacillus. Both of them achieved reduction, however, only Melo et al. ${ }^{13}$ had a significant reduction.

Several PSs are used to achieve the antimicrobial effect during the application of aPDT, but MB and TBO have been the most commonly tested option in in vivo and in situ studies. $^{20,23-25}$ In this context, it is important to analyze the absorption spectrum of the PS and the emission spectra of the light source to have an efficient photodynamic action. Guglielmi et al. ${ }^{10}$ and Steiner-Oliveira et al., ${ }^{14}$ in one of their experimental groups, used MB and red low power LASER light source with a wavelength of $660 \mathrm{~nm}$ that is coincident with the absorption band of MB (610 to $660 \mathrm{~nm}) .{ }^{26}$ Araújo et al. ${ }^{12}$ also used $\mathrm{MB}$, however, a halogen curing light unit was used, which emits white light that has an emission spectrum ranging between 500 and $800 \mathrm{~nm}$. Melo et al. ${ }^{13}$ and Borges et al. $^{9}$ used TBO and LED sources that provided the emission spectrum within the characteristic absorption range for this PS (590 to $630 \mathrm{~nm}) .{ }^{26}$ Longo et al., ${ }^{11}$ in turn, used AlClPc and a red laser of $660 \mathrm{~nm}$, which is coincident with the electromagnetic spectrum, in which the phthalocyanine group absorbs light (660 to $700 \mathrm{~nm}) .{ }^{26}$ However, Steiner-Oliveira et al. ${ }^{14}$ tested two PS s, TBO with LED and MB with a low-power laser. This study demonstrated that all therapies, including the control group, reduced the number of all tested microorganisms except for S. sobrinus, and no statistical differences were observed among the protocols. In turn, there are no differences between the LED and low-power laser parameters. For these authors, the main goal of aPDT is to perform a conservative treatment of deep caries lesions to reduce the number of microorganisms in the remaining affected dentin to avoid the need for endodontic treatment.

The preirradiation time of $5 \mathrm{~min}$ appears to be an important detail to reach the antibacterial effect of aPDT PS in regards to maintaining the inside bacteria, and this time enables greater absorption of light. ${ }^{27}$ Borges et al. ${ }^{9}$ showed that the use of a laser without a PS, and PS $\mathrm{s}$ in the absence of a light source, result in a minimal reduction in the amount of bacteria, without a significant effect on the reduction of microorganisms.

With regard to the volume of dentin analyzed, only Borges et al. ${ }^{9}$ and Melo et al. ${ }^{13}$ provided the dentin weight information. The weighing of each dentin can promote the standardization of the number of CFU/mg of dentin collected, and thus enables better comparisons. Based on this, we recommend that future studies weigh the dentin and evaluate the amount of CFU/mg of dentin that is analyzed.

Of all of the studies that used CFU as their counting method and to which we had access to the data, the study of Araújo et al. ${ }^{12}$ stood out for achieving the highest log reduction value.

This systematic review has grouped the data found in the selected studies, describing the parameters of the aPDT, and the species that each of the studies assessed. It was observed 
that, in the studies included in this review, the vast majority of bacteria investigated were of the Gram-positive genus, which are microorganisms likely to be affected by aPDT. This can be explained on the basis of the link mechanism of cell membrane, in a function of the structural variations in their cell walls. Gramnegative bacteria have an outer membrane complex that includes two lipid bilayers that serve as a physical and functional barrier between the cells and the environment, whereas Gram-positive cells are more sensitive and have a relatively permeable thickness of membrane. ${ }^{28}$

While analyzing the aPDT parameters used in the selected studies, we observed that different culture media and atmospheres for incubating the microorganisms were used. In relation to the way samples were transported to the laboratory for $\mathrm{CFU}$ analyses, Guglielmi et al. ${ }^{10}$ used the VMGA III, a transport media, Longo et al. ${ }^{11}$ selected BHI medium, whereas Araújo et al. ${ }^{12}$ performed immediate processing. Borges et al. ${ }^{9}$ and Melo et al. ${ }^{13}$ did not provide this information. All specimens must be promptly transported to the laboratory for CFU counts, preferably within $2 \mathrm{~h}$. If processing is delayed, specimens collected for detection of bacterial agents may be stored under specified conditions. ${ }^{29}$ Generally, transport media provide a nonnutrient source that sustains the viability of both aerobic and anaerobic organisms without allowing significant growth. ${ }^{30}$ The BHI medium, used by Longo et al. ${ }^{11}$ as a transport media, is a nutritive base that is used to cultivate a wide variety of organisms. ${ }^{31}$ This medium can overestimate the number of CFU.

The samples that were analyzed by quantitative real-time PCR were placed in microtubes and stored at $-80^{\circ} \mathrm{C}$ until used $^{12}$ or placed in microtubes containing $100 \mu \mathrm{L}$ of TE buffer and stored at $-20^{\circ} \mathrm{C}$ until used. ${ }^{14}$

Although the results of these studies are encouraging, more in vivo studies are necessary to solve doubts regarding parameters such as irradiation time and whether the use of multiple irradiation points improves the use of this therapy in clinical settings.

\section{Conclusion}

Our study demonstrated that aPDT is an effective coadjuvant therapy to reduce microorganisms in deep carious lesions.

\section{Acknowledgments}

The authors would like to thank the authors of the selected articles for the contribution of available data from their papers. We are indebted to Sarah Vinski who revised the text for grammar and style. This manuscript is part of the first author's dissertation. L.A.A. was supported by Carlos Chagas Filho Foundation for Research Support of Rio de Janeiro State (FAPERJ-Jovem Cientista do Nosso Estado). The authors declare no potential conflicts of interest with respect to the authorship and/or publication of this article.

\section{References}

1. P. Frãzao, "Epidemiology of dental caries: when structure and context matter," Braz. Oral Res. 26(Suppl 1), 108-114 (2012).

2. A. T. Hara and D. T. Zero, "The caries environment: saliva, pellicle, diet, and hard tissue ultrastructure," Dent. Clin. North Am. 54(3), 455-467 (2010).

3. F. Schwendicke et al., "Failure of incompletely excavated teeth-a systematic review," J. Dent. 41(7), 569-580 (2013).
4. D. S. Wambier et al., "Ultrastructural and microbiological analysis of the dentin layers affected by caries lesions in primary molars treated by minimal intervention," Pediatr. Dent. 29(3), 228-234 (2007).

5. B. Azrak et al., "Comparison of the efficacy of chemomechanical caries removal (Carisolv) with that of conventional excavation in reducing the cariogenic flora," Int. J. Paediatr. Dent. 14(3), 182-191 (2004).

6. L. J. Motta et al., "Efficacy of Papacarie(®) in reduction of residual bacteria in deciduous teeth: a randomized, controlled clinical trial," Clinics 69(5), 319-322 (2014).

7. H. Gursoy et al., "Photodynamic therapy in dentistry: a literature review," Clin. Oral Invest. 17(4), 1113-1125 (2013).

8. R. A. Arneiro et al., "Efficacy of antimicrobial photodynamic therapy for root canals infected with Enterococcus faecalis," J. Oral Sci. 56(4), 277-285 (2014).

9. F. M. C. Borges et al., "Evaluation of the effect of photodynamic antimicrobial therapy in dentin caries: a pilot in vivo study," Proc. SPIE 7549, 75490B (2010).

10. C. de A. B Guglielmi et al., "Clinical use of photodynamic antimicrobial chemotherapy for the treatment of deep carious lesions," J. Biomed. Opt. 16(8), 088003 (2011).

11. J. P. Longo et al., "Photodynamic therapy disinfection of carious tissue mediated by aluminum-chloride-phthalocyanine entrapped in cationic liposomes: an in vitro and clinical study," Lasers Med. Sci. 27(3), 575-584 (2012).

12. P. V. Araújo et al., "Antimicrobial effect of photodynamic therapy in carious lesions in vivo, using culture and real-time PCR methods," Photodiagn. Photodyn. Ther. 12(3), 401-407 (2015).

13. M. A. Melo et al., "Photodynamic antimicrobial chemotherapy and ultraconservative caries removal linked for management of deep caries lesions," Photodiagn. Photodyn. Ther. 12(4), 581-586 (2015).

14. C. Steiner-Oliveira et al., "Randomized in vivo evaluation of photodynamic antimicrobial chemotherapy on deciduous carious dentin," J. Biomed. Opt. 20(10), 108003 (2015).

15. L. A. Stewart et al., "PRISMA-IPD development group. preferred reporting items for systematic review and meta-analyses of individual participant data: the PRISMA-IPD statement," J. Am. Med. Assoc. 313(16), 1657-65 (2015).

16. J. P. Higgins et al., "Cochrane bias methods group; cochrane statistical methods group. The cochrane collaboration's tool for assessing risk of bias in randomised trials," Br. Med. J. 343, d5928 (2011).

17. P. T. J. Higgins and S. Green, "Cochrane handbook for systematic reviews of interventions. Version 5.1.6," The Cochrane Collaboration (2011) www.cochrane-handbook.orgurisimplewww.cochrane-handbook. org (15 January 2016).

18. C. C. Tonon et al., "Comparative effects of photodynamic therapy mediated by curcumin on standard and clinical isolate of Streptococcus mutans," J. Contemp. Dent. Pract. 16(1), 1-6 (2015).

19. M. C. Borsatto et al., "One-session root canal treatment with antimicrobial photodynamic therapy (aPDT): an in vivo study," Int. Endod. J. 49(6), 511-518 (2016).

20. J. P. Lima et al., "Evaluation of the antimicrobial effect of photodynamic antimicrobial therapy in an in situ model of dentine caries," Eur. J. Oral Sci. 117(5), 568-574 (2009).

21. I. M. Diniz et al., "Antimicrobial photodynamic therapy: a promise candidate for caries lesions treatment," Photodiagn. Photodyn. Ther. 12(3), 511-518 (2015)

22. D. Moher et al., "Consolidated standards of reporting trials group. CONSORT 2010 explanation and elaboration: updated guidelines for reporting parallel group randomised trials," J. Clin. Epidemiol. 63(8), e1-e37 (2010).

23. M. A. S. Melo et al., "In vitro photodynamic antimicrobial chemotherapy in dentine contaminated by cariogenic bacteria," Laser Phys. 20, 1504-1513 (2010).

24. M. A. Monteiro-Oliveira et al., "Photodynamic therapy effect in carious bovine dentin — an in vitro study," J. Oral Laser Appl. 10(1), 29 (2010).

25. A. H. Teixeira et al., "Effect of photodynamic antimicrobial chemotherapy on in vitro and in situ biofilms," Caries Res. 46(6), 549-554 (2012).

26. C. P. Sabino and M. S. Ribeiro, "Dosimetria na terapia fotodinâmica antimicrobiana," Chapter 3, in PDT: Terapia Fotodinâmica Antimicrobiana na Odontologia, S. C. Núñez, M. S. Ribeiro, and A. S. Garcez, pp. 27-38, Elsevier, Rio de Janeiro, Brasil (2013). 
27. T. Ito, "Toluidine blue: the mode of photodynamic action in yeast cells," Photochem. Photobiol. 25(1), 47-53 (1977).

28. J. Y. Nagata et al., "Antibacterial photodynamic therapy for dental caries: evaluation of the photosensitizers used and light source properties," Photodiagn. Photodyn. Ther. 9(2), 122-131 (2012).

29. J. M. Miller, K. Krisher, and H. T. Holmes, "General principles of specimen collection and handling," Chapter 5 in Manual of Clinical Microbiology, P. R. Murray et al., Eds., pp. 43-54, ASM Press, Washington (2007).

30. K. C. Chapin, "Principles of stains and media," Chapter 14 in Manual of Clinical Microbiology, P. R. Murray et al., Eds., pp. 182-191, ASM Press, Washington (2007).

31. K. C. Chapin and T. Lauderdale, "Reagents, stains, and media: bacteriology," Chapter 21 in Manual of Clinical Microbiology, P. R. Murray et al., Eds., pp. 334-364, ASM Press, Washington (2007).

Pâmela Oliveira Ornellas is an MSc student at the School of Dentistry, Fluminense Federal University, Nova Friburgo, Rio de Janeiro, Brazil. Her main topic of research interest is the effect of the antimicrobial photodynamic therapy on microorganism reduction in deep caries lesions.

Leonardo Santos Antunes is an assistant professor in endodontic clinic at Fluminense Federal University, Nova Friburgo, Rio de Janeiro, Brazil. He received his MSc degree in dentistry in 2006 and his $\mathrm{PhD}$ in science in 2012 at Fluminense Federal University, Niterói, Rio de Janeiro, Brazil. His research interests are basically related to the applications of photodynamic therapy in dentistry, endodontic field, and dental genetics.

Karla Bianca Fernandes da Costa Fontes received her MSc and $\mathrm{PhD}$ degrees in oral pathology from the Fluminense Federal University, Niterói, Rio de Janeiro, Brazil, in 2007 and 2009, respectively. Currently, she is working as an assistant professor for oral pathology and oral medicine at Fluminense Federal University, Nova Friburgo, Rio de Janeiro, Brazil. Her research interests are basically related to the applications of photodynamic therapy and laser therapy in dentistry.

Helvécio Cardoso Corrêa Póvoa is an assistant professor for microbiology at Fluminense Federal University, Nova Friburgo, Rio de Janeiro, Brazil. He received his MSc degree in microbiology in 1999 from the University State of Rio de Janeiro, Rio de Janeiro, Brazil and his PhD in patology in 2008 from Fluminense Federal University, Niterói, Rio de Janeiro, Brazil. His research interests are basically related to the applications of photodynamic therapy in microbiology.

Erika Calvano Küchler is dental researcher in pediatric dentistry at the University of São Paulo, School of Dentistry of Ribeirão Preto, São Paulo, Brazil. She received her MSc degree in pediatric dentistry in Rio de Janeiro Federal University, Rio de Janeiro, Brazil and her PhD in science in Fluminense Federal University, Niterói, Rio de Janeiro, Brazil. Her research focuses are related to molecular and clinical dental research.

Natalia Lopes Pontes lorio received her MSc and PhD degrees in microbiology from Rio de Janeiro Federal University, Rio de Janeiro, Brazil, in 2007 and 2011, respectively. Currently, she is working as an assistant professor for microbiology at Fluminense Federal University, Nova Friburgo, Rio de Janeiro, Brazil. Her research interests are basically related to the applications of photodynamic therapy in microbiology.

Lívia Azeredo Alves Antunes received her MSc and $\mathrm{PhD}$ degrees in pediatric dentistry from Rio de Janeiro Federal University, Rio de Janeiro, Brazil in 2006 and 2012, respectively. Currently, she is working as an assistant professor for pediatric dentistry at Fluminense Federal University, Nova Friburgo, Rio de Janeiro, Brazil. Her research interests are related to the applications of photodynamic therapy in dentistry, minimally invasive dentistry, quality of life, and dental genetics. 